\title{
THE SYNTACTIC AND SEMANTIC PROPERTIES OF MODAL WORDS IN GERMAN
}

\author{
Anna Averina \\ Moscow State Region University, \\ ul. Radio 10A, 105005, Moscow, Russia
}

\begin{abstract}
The aim of the article is to examine the semantic and syntactic properties of modal words in German. The paper analyzes the types of modal semantics which are encoded in modal words; the peculiarities of the syntactic use of modal words are shown; the relationship between the type of modal semantics and their syntactic use is revealed, and the question which words with evaluative semantics can be classified as modal is considered. The relevance of this kind of work is determined by the following circumstances: firstly, it is necessary to describe the syntax and the semantics of modal words and their grammar features; secondly, it is important to reveal the relationship between modality and evaluativity and, thirdly, to define the conditions under which modal words can encode not one but several modal meanings. By using the transformation method and the method of component analysis the following groups of modal words were identified: modal words with alethic semantics; modal words with epistemic semantics; modal words with evidential semantics. Some modal words build separate groups: The modal word angeblich has evidential semantics, because it indicates the third person as a source of information; the modal word wahrscheinlich can have epistemic or evidential or alethic and evidential semantics; the modal word leider is able to express an emotional attitude of the speaker. The identification of different types of modal words is possible through the research of their syntactic properties, namely: the usage in dependent object clauses with the epistemic and factive predicate in the matrix clause; in conditional sentences as well as the ability to have independent usage as an answer to a question without a question word.
\end{abstract}

Key Words: modal words, alethic modality, evaluation, epistemicity, evidentiality, modal proposition

For citation: Averina A.V. 2021. The Syntactic and Semantic Properties of Modal Words in German. Philological Sciences at MGIMO.Vol. 7. No 1(25). P. 5-14. https://doi.org/10.24833/2410-2423-20211-25-5-14

\section{СИНТАКСИС И СЕМАНТИКА МОДАЛЬНЫХ СЛОВ В НЕМЕЦКОМ ЯЗЫКЕ}

\author{
А.В. Аверина \\ Московский государственный областной университет, \\ 141014, Московская область, г. Мытищи, ул. Веры Волошиной, д. 24
}


Аннотация. Цель настоящей статьи состоит в описании семантических и синтаксических свойств модальных слов в немеиком языке. В работе проанализированы виды модальной семантики, передаваемой модальными словами; показаны особенности синтаксического употребления модальных слов; раскрыта взаимосвязь между видом модальной семантики и их синтаксическим употреблением, а также рассмотрен вопрос о том, какие слова с оценочной семантикой можно отнести к разряду модальных. Актуальность работь подобного рода определяется, вопервых, необходимостью описания семантики и синтаксиса модальных слов и, соответственно, их грамматических свойств; во-вторьх, потребностью в выявлении соотночения между модальностью и оценочностью; в-третьих, важностью определения условий, при которьх модальные слова могут нести не одно, а два модальных значения. Посредством использования метода трансформации и метода компонентного анализа были выделены следующие группь модальных слов: модальные слова с алетической семантикой; модальные слова с эпистемической семантикой; модальные слова с эвиденииальной семантикой. Некоторые модальные слова образуют отдельные подгруппь: модальное слово angeblich несёт эвиденииальную семантику, поскольку оно указывает только на 3-е лицо как на источник информации; модальное слово wahrscheinlich может иметь эпистемическую, эвиденииальную или алетическую семантику; модальное слово leider способно передавать эмоциональную оценку с позиции говорящего. Выявление различных типов модальных слов стало возможным благодаря исследованию их синтаксических свойств, а именно: использование в придаточных объектных при эпистемическом и при фактивном предикате в главном предложении; в придаточных условных, а также способность иметь самостоятельное употребление при ответе на вопрос без вопросительного слова.

Ключевые слова: модальные слова, алетическая модальность, оценочность, эпистемичность, эвиденииальность, модализованная пропозиция

Для цитирования: Аверина А.В. 2021. Синтаксис и семантика модальных слов в немецком языке. Филологические науки в МГИМО. Том 7. № 1(25). С. 5-14. https://doi.org/10.24833/24102423-2021-1-25-5-14

\section{1. Введение}

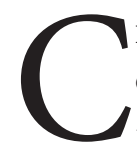

истема частей речи немецкого языка включает в себя такой разряд слов как модальные слова. Для их обозначения используют термины «модальные слова» (Modalwörter) [9; 12], «модальные наречия» (Modaladverbien [8] или Modalitätsadverbien [17]); «наречия, относящиеся ко всему предложению» (Satzadverbien [20]). Обращает на себя внимание тот факт, что модальные слова неоднородны по своей семантике, что, в свою очередь, влияет на особенности их синтаксического употребления. Это позволяет говорить о том, что они представляют собой дифференцированный класс слов. Так, если сопоставить возможности их включения в придаточное предложение места (1), то можно заметить, что они обладают различными свойствами модальное слово vielleicht можно рассматривать как синтаксически неподчинимое, в то время как модальное слово tatsächlich может иметь место в структуре придаточного предложения:

(1) Das Öl kommt also genau dorthin, wo es ( ${ }^{*}$ vielleicht, + tatsächlich $\left.{ }^{1}\right)$ sein soll [10].

Цель настоящей работы состоит в том, чтобы описать семантические и синтаксические особенности класса модальных слов в немецком языке. Цель определила следующие задачи: (1) проанализировать виды модальной семантики, передаваемой модальными словами; (2) показать особенности синтаксического употребления модальных слов; (3) раскрыть взаимосвязь между видом модальной семантики и особенностями их синтаксического употребления и (4) рассмотреть вопрос о том, какие слова с оценочной семантикой можно отнести к разряду модальных.

\footnotetext{
1 Здесь и далее: знаком $\left(^{*}\right)$ обозначены случаи, в которых включение модального слова невозможно, знак (+) служит для того, чтобы обозначить возможные допустимые варианты.
} 
Актуальность настоящей работы определяется, во-первых, необходимостью описания семантики и синтаксиса модальных слов и, соответственно, их грамматических свойств; во-вторых, потребностью в выявлении соотношения между модальностью и оценочностью; в-третьих, важностью определения условий, при которых модальные слова могут нести не одно, а два модальных значения. В качестве материалов исследования послужили примеры, отобранные из немецкого веб-корпуса DECOW 16A и примеры из немецкой художественной литературы. Выбор художественных текстов объясняется тем, что в некоторых случаях для выявления семантических особенностей модальных слов был необходим анализ более широкого контекста. Всего было отобрано и проанализировано свыше 200 примеров.

\section{2. Семантика модальных слов и их синтаксические свойства}

\section{1. Модальные слова в системе частей речи}

Модальные слова некоторые исследователи относят к разряду наречий [4], однако большинство лингвистов склоняются к тому, что модальные слова отличаются от наречий по следующим параметрам (обзор представлен в [5, с. 426]:

a) не выступают в качестве отдельного члена предложения [6, с. 367-368];

б) относятся не к отдельному члену предложения, а ко всему высказыванию в целом [6, с. 368];

в) могут оформлять общевопросительные предложения (Kommst du? - Vielleicht), в то время как наречия могут оформлять только частновопросительные предложения («Wie singt Klara?»«Schön») $[9$, c. $203 ; 5$, c. 426-427].

Перечисленные свойства в разной степени проявляются у модальных слов с различными видами модальных значений. Так, модальные слова, кодирующие эпистемичность, могут выступать в самостоятельном употреблении в роли ответа на вопрос, что нехарактерно для модальных слов с эвиденциальной и алетической семантикой, сравним:

(2a) Kommst du? *Angeblich.

(2b) Kommst du? Vielleicht.

(2c) Kommst du? ${ }^{*}$ Tatsächlich.

В роли самостоятельного ответа на вопрос не может быть использовано и слово с оценочной семантикой leider:

(2d) Kommst du? ${ }^{\star}$ Leider.

Остановимся подробнее на характеристике типов модальных значений, выражаемых модальными словами.

2.2. Типы модальных значений, передаваемых модальными словами, и критерии их разграничения

Модальная семантика многопланова: в немецких грамматиках принято говорить об эпистемических и не-эпистемических значениях [22, с. 1884-1885]. К не-эпистемическим значениям относят волюнтативное (3), деонтическое (4), алетическое (5) значения, например:

(3) Er will sich Gedanken machen, wie man das lösen könnte [10] (выражение волеизъявления - волюнтативная модальность);

(4) Da muss man mit der Zeit gehen [10] (выражение долженствования - деонтическая модальность);

(5) Da kann jeder Kundenstimmen abgeben [10] (выражение потенциальной возможности - алетическая модальность).

К эпистемическим значениям можно отнести семантику предположения и эвиденциальную семантику: речь идёт о пропозициональной установке говорящего / потенциального говорящего, выражаемой по отношению к содержанию высказывания, например:

(6) Vielleicht hat er einfach nur seine echte schlechte Laune zur Schau gestellt [10]. (Выражение предположения, т.е. эпистемическая модальность).

(7) Es musste altes Militär dabei sein, das durch die Marschmusik angeregt wurde - denn machtvoll erhob sich nach kurzer Zeit das unvergängliche Lied [...] [21]. (Выражение предположения + семантика инференциальности, относящаяся к сфере эвиденциальных значений). 
Модальные слова в немецком языке способны передавать эпистемическое, эвиденциальное и алетическое значения, в некоторых случаях одно и то же модальное слово меняет тип модальной семантики в зависимости от контекстуального окружения. Рассмотрим в этой связи примеры, в которых модальное слово передаёт только одно модальное значение:

(8) Vielleicht wären 5er Trupps bei Dow stylischer gewesen, andersrum ist es fraglich das es Sinn macht sich im Feld als strikte Größen zu halten [10] - модальное слово vielleicht служит для передачи эпистемической семантики;

(9) Tatsächlich sind hier nicht einfach ein paar Szenen zugefügt oder entfernt worden, sondern der Film kommt in der überarbeiteten Version extrem deutlich verändert rüber [10] - модальное слово tatsächlich служит для передачи алетической семантики;

(10) Angeblich wurden keine anderen Fehler gefunden und man geht davon aus daß die Festplatten defekt sind [10] - модальное слово angeblich служит для передачи эвиденциальной семантики.

Э. Лайсс обращает внимание на то, что модальные слова не способны передавать два значения одновременно (например, значение эпистемичности и эвиденциальности), как это возможно в случае с модальными глаголами [16]. Она связывает это с тем, что им свойственна меньшая степень грамматикализованности: семантика модального слова может быть интерпретирована вне контекста. Так, например, модальные слова angeblich, scheinbar и anscheinend передают эвиденциальные значения, модальные слова vielleicht, vermutlich, wahrscheinlich и sicher - эпистемические. Модальные слова немецкого языка Э. Лайсс интерпретирует как одноплановые дейктики, функция которых состоит в указании на нефактичность ситуации [Там же]. Действительно, модальные слова типа anscheinend и scheinbar участвуют в передаче эвиденциальных значений, указывая на источник информации, а модальные слова vielleicht, vermutlich - в выражении эпистемической семантики, сравним:

(11) Wer weiss, vielleicht kommts ja noch [10].

(12) Gardner weiß anscheind selber nicht mehr, wo ihm der Kopf steht [10].

В некоторых случаях в высказывании с модальными словами сложно определить, идёт ли речь об алетической модальности (потенциальной возможности) или об эпистемической (значении предположения). Так, например, М. Хундт указывает на то, что предложение (13) может иметь двоякое толкование:

(13) Möglicherweise regnet es heute.

Оно может быть интерпретировано как предложение с алетико-фактической или эпистемической модальностью: с одной стороны, указывается на возможность того, что пойдёт дождь, с другой стороны, говорящий высказывает своё предположение относительно того, что может пойти дождь $[14$, S. 252]. Основное отличие между двумя различными интерпретациями состоит в том, что первая предполагает существование двух пропозиций, вторая - существование одной пропозиции и пропозициональной установки [Там же, S. 370]. В первом случае предложение можно парафразировать так: ,Ich als Sprecher behaupte, dass folgendes der Fall ist'. В случае с эпистемической модальностью требуется иная парафраза: ,Ich als Sprecher halte es für möglich, dass gilt [...]' [Там же]. Из этого следует, что в первом случае речь идёт об утверждении (ассерции).

Модальные слова wirklich и tatsächlich служат для выражения утверждения, которое имеет не эпистемическую, а алетическую интерпретацию. Это можно проследить на следующих примерах:

(14) „Er geht an Braumüller 'ran! Himmelherrgott, heiliger Moses!“ schrie Lenz jetzt, „er ist tatsächlich vorbei und geht an Braumüller 'ran" [21] (модальное слово tatsächlich служит для утверждения истинности позиции говорящего);

(15) Aber der Wagen ist wirklich zu teuer [21] (истинность позиции говорящего передаёт модальное слово wirklich).

Рассмотрим модальную семантику слова wahrscheinlich. Как показывает анализ фактического материала, оно участвует в передаче эвиденциальных значений, поскольку предположение говорящего базируется на его умозаключении, а выводы, базирующиеся на умозаключении, входят в семантическую зону эвиденциальных значений (см. [7]). Рассмотрим примеры: 
(16) Du musst nur deutlich aufmerksamer und konzentrierter sein. Wahrscheinlich hast du keinen Blick auf die Links geworfen [10].

(17) Wahrscheinlich sind wir da sogar aneinander vorbeigefahren, da ich am selben Tag ebenfalls dort war [10].

В приведённых высказываниях речь идёт об инференциальной семантике как разновидности эвиденциальных значений. Это позволяет считать, что модальное слово wahrscheinlich способно передавать эпистемичность или эвиденциальность.

Заслуживают внимания и такие предложения как:

(18a) Hier war wahrscheinlich die erste Siedlung [10].

(19a) Es war wahrscheinlich auch Ziel des Varus [10].

Данные предложения можно интерпретировать как передающие семантику алетической модальности, так и как предложения с эпистемической модальностью. При решении вопроса о целесообразности отнесения модального маркера к сфере эпистемических/эвиденциальных/ алетических значений можно воспользоваться критериями, предложенными Д. Лайонзом: (1) проследить совместимость модальных маркеров с фактивными глаголами и (2) проследить способность модального маркера быть в скопусе показателей эпистемической модальности: алетические значения могут быть в скопусе эпистемических ${ }^{2}$ [19].

Согласно этим критериям модальные слова wirklich и tatsächlich действительно передают алетическую модальность, поскольку они способны иметь место в объектных придаточных предложениях с фактивными предикатами в главной части, например:

(20) Er bezweifelt, dass der Wagen TATSÄCHLICH vorbei ist.

(21) Er bezweifelt, dass der Wagen WIRKLICH zu teuer ist.

В приведённых высказываниях модальные слова tatsächlich и wirklich несут фокусный акцент. Как известно, функция фокусного выделения состоит в выражении одной из альтернатив ${ }^{3}[18$, S. 102]. Таким образом, модальные слова wirklich и tatsächlich можно рассматривать как фокусные экспоненты (в немецкой терминологии - Fokusexponenten), они подчёркивают одну из альтернатив, которая существуют в объективной действительности. При этом кодируется контрастивный топик, или Verumfokus (подробнее см. [13]).

Рассмотрим семантику модального слова wahrscheinlich. В корпусе не было найдено примеров, на которых можно было бы проследить возможности его включения в придаточное объектное с фактивным предикатом в главной части. По мнению носителей языка, в виде исключения это возможно в том случае, если модальное слово wahrscheinlich относится к одному члену предложения, например:

(18b) Er bezweifelt, dass wahrscheinlich HIER die erste Siedlung war.

В приведённом высказывании модальное слово wahrscheinlich служит для фокусировки наречия места hier. Аналогичную ситуацию это можно проследить и на следующем примере:

(19b) Sie bezweifelt, dass es wahrscheinlich AUCH das Ziel des Varus war.

Это позволяет заключить, что модальное слово wahrscheinlich в определённых условиях может передавать алетическое значение, что нехарактерно для модальных слов vielleicht, vermutlich, scheinbar, сравним:

(18c) Sie bezweifelt, dass $\left({ }^{*}\right.$ vermutlich, ${ }^{*}$ vielleicht, ${ }^{*}$ scheinbar) hier die erste Siedlung war.

Перейдём к анализу модальных слов немецкого языка по второму критерию: маркеры алетической модальности находятся в скопусе маркеров субъективной модальности. Модальное слово wahrscheinlich вполне может сочетаться с маркерами эпистемической модальности, поскольку обнаруживает сходство с ними. Так, например, оно довольно употребимо в предложениях с частицами:

\footnotetext{
В оригинале: „Die Objektivierung der epistemischen Modalität ist eine Voraussetzung dafür, dass wir über vergangene oder zukünftige Möglichkeiten (cf. Gestern bestand eine gewisse Möglichkeit, dass es heute regnen würde) reden können und dass eine epistemische Modalität im Skopus einer zweiten auftritt (cf. Es ist möglich, dass es notwendig ist, dass ...)" [19, S.405].

3 „Sinn der starken Akzentuierung ist dann, vom Kontext her mögliche Alternativen auszuschließen“ [18, S. 102].
} 
(22) Dass es die SV 650 auch vollverschalt gibt, weißt du ja wahrscheinlich ... [10].

(23) [...] sie hat wohl wahrscheinlich an eine[r] Gardine geknabbert [10].

Особенность слова wahrscheinlich состоит в том, что оно может выступать в роли наречия: перед ним могут стоять усилительные частицы sehr, ziemlich, например:

(24) Mein morgiger Flug von HAM nach MUC wird wohl sehr wahrscheinlich nicht stattfinden [6].

(25) Das wäre ziemlich wahrscheinlich ein wahrer Aussagesatz [10].

В приведённых высказываниях слово wahrscheinlich выступает больше для выражения онтологической возможности.

Итак, анализ фактического материала показывает, что модальное слово wahrscheinlich может передавать эпистемическое, эвиденциальное и алетическое значения.

Совершенно однозначно модальные слова wirklich и tatsächlich могут быть в скопусе маркеров эпистемической модальности:

(26) Hier dürfte wirklich der Faktor Glück die entscheidene Rolle spielen!!! [10];

(27) Es kann aber natürlich auch sein, dass sie tatsächlich ein Hinweis auf ganz neue Phänomene sind! [10].

Это ещё раз подтверждает тот факт, что они передают алетическую семантику.

Особое место в системе модальных слов немецкого языка занимает слово leider - оно не относится ни к эпистемическим, ни к эвиденциальным маркерам, ни к показателям алетической модальности. Речь идёт скорее о выражении оценочности. Слова с оценочной семантикой в немецком языкознании не относят к системе модальных значений. Напротив, в отечественных исследованиях оценка зачастую рассматривается как разновидность модальных значений. ${ }^{4}$ Ранее анализ данного вопроса подвёл нас к выводу о том, что оценочность входит в модальную сферу в том случае, если речь идёт о целостном тексте и модальности текста [1, с. 16-17]. Оценочные наречия типа erstaunlicherweise, erfreulicherweise, interessanterweise отражают эмоциональное состояние субъекта:

(28a) Erstaunlicherweise unterschied sich die weibliche Kleidung nur geringfügig von der männlichen [10].

(29a) Leider hat der Film auch eine tragische, sehr traurige Seite [10].

(30) Erfreulicherweise zog auch bei allen Unternehmen das Neugeschäft an [10].

Специфика их состоит в том, что они могут относиться к целому предложению, как это видно на примерах (28) - (30). Как показывает трансформационный анализ, некоторые слова с данной семантикой не могут быть в скопусе показателей эпистемической модальности, например:

$\left(28 \mathrm{~b}^{*}\right)$ Er vermutet, dass sich (bedauerlicherweise, erfreulicherweise) die weibliche Kleidung nur geringfügig von der männlichen unterschied.

$\left(29 b^{*}\right)$ Er vermutet, dass der Film leider eine tragische, sehr traurige Seite hat.

Напротив, наречия с семантикой оценки типа ratsamerweise, sonderbarerweise могут быть в скопусе показателей эпистемической модальности, сравним:

(31a) Er vermutet, dass der Film sonderbarerweise gedreht wurde [10].

(32a) Er vermutet, dass diese Arbeit ratsamerweise zu erledigen ist [10].

Это может иметь двоякое объяснение: во-первых, они относятся не ко всему предложению, а к какому-либо его компоненту, а, во-вторых, служат не для оценки эмоционального состояния субъекта, а для характеристики какого-либо действия.

Наречия, обозначающиеэмоциональноесостояниетипаleider, erstaunlicherweise, erfreulicherweise, interessanterweise, не могут сочетаться с фактивными предикатами (см. (28c) и (29c)), в то время как наречия ratsamerweise, sonderbarerweise, указывающие не на эмоциональное состояние субъекта, а на качественную оценку действий / событий / объектов, - могут (см. (33)-(34)), например:

\footnotetext{
4 Так, Е.М. Вольф пишет: «Оценку можно рассматривать как один из видов модальностей, которые накладываются на дескриптивное содержание языкового выражения ... оценочная модальность определяется высказыванием в целом, а не отдельными его элементами, и является компонентом высказывания» [3, с. 11].
} 
$\left(28 c^{\star}\right)$ Er leugnet, dass sich bedaurlicherweise die weibliche Kleidung nur geringfügig von der männlichen unterschied.

$\left(29 c^{*}\right)$ Er leugnet, dass der Film leider eine tragische, sehr traurige Seite hat.

(31b) Er leugnet, dass der Film sonderbarerweise gedreht wurde.

(32b) Er leugnet, dass diese Arbeit ratsamerweise zu erledigen ist.

Для того, чтобы точно определить, входит ли семантика слов с оценочной семантикой в пропозициональное содержание, может быть информативным следующий тест: включение их в придаточное условия. По наблюдениям Д. Лайонза, всё, что входит в скопус кондиционального оператора, является частью пропозиционального содержания [19, S. 404-405]. Это позволяет ему объяснить тот факт, почему модальные глаголы теряют вторичное значение в придаточном условном. Это связано, по всей видимости, с тем, что речь идёт об особом типе модальности, кодированном в данном типе предложения - об «условной фактичной, нефактичной и контрфактичной модальности» (М.Л. Котин) („konditionale faktische, nichtfaktische und kontrafaktische Modalität" $[15$, S. 143]), не совместимой с эпистемическими маркерами. Условные придаточные включают в себя описание не событий, а условий. Как отмечает Т. Фритц, немодализованные, мыслимые факты являются базой для модализованных, утверждаемых фактов [11]. То, что утверждается, нельзя предполагать.

Анализ фактического материала показывает, что модальные слова wirklich и tatsächlich могут быть в придаточных предложениях условиях, так как кодируют алетическую модальность, а она входит в пропозицию предложения (речь идёт о модализованной пропозиции - см. Т.В. Булыгина, А.Д. Шмелёв [2]), в то время как модальные слова vielleicht, vermutlich и т. д. не могут, так как передают эпистемическую семантику, не входящую в пропозициональное содержание, сравним:

(33) Heizenergie soll dannfür den Verbrauch zur Verfügung stehen, wenn sie tatsächlich ( ${ }^{*}$ wahrscheinlich, ${ }^{*}$ vielleicht, ${ }^{*}$ vermutlich, +wirklich) benötigt wird [10].

Модальное слово angeblich может быть включено в придаточные условия: как правило, в роли субъекта выступает 3-е лицо:

(34) Merken Sie eigentlich nicht, wie dumm das ist? Wenn angeblich ( ${ }^{*}$ scheinbar, ${ }^{\star}$ anscheinend) schon die Grundlagen falsch sind, dann können Sie Kapitel 4 gleich mit wegschmeißen [10].

Вместо модального слова angeblich не могут быть использованы слова scheinbar и anscheinend. По всей видимости, это связано с тем, что angeblich указывает на 3-е лицо как на источник информации, что сближает его с пропозициональным содержанием предложения, а не с пропозициональной установкой, в то время как использование scheinbar и anscheinend предполагает, что в роли источника информации выступает 1-е лицо и предложение приобретает субъективную оценку с позиции говорящего.

Анализ фактического материала показывает, что слово с оценочной семантикой leider в большинстве случаев может иметь место в придаточном условия. Если в предложении есть ограничители типа nur, genau, сужающие скопус этого слова, то его включение в придаточное возможно:

(35) Aber an dieser Stelle macht Ihr den Fehler, wenn Ihr leider genau dieses Modell hattet [10].

Это возможно и в том случае, если придаточное эллиптично. В корпусе можно встретить примеры типа:

(36) Wenn leider nein, sende ich dir ganz, ganz herzliche Grüße und viel Kraft [10].

В корпусе есть примеры, демонстрирующие возможность включения слова leider в придаточное условия, если оно содержит иные модальные маркеры, например:

(37) Wenn es leider zu solchen Einsätzen kommen sollte, dann haben wir im 1. LF eine Spielzeugkiste wo sich die Kinder dran Austoben können [10].

В приведённом высказывании модальный глагол sollen участвует в передаче деонтической семантики.

В случаях, если сложное предложение содержит объективированное изложение событий, включение слова leider в придаточное условное вряд ли будет корректным:

(38) [...] wenn an einem Unternehmen (?leider) viele Menschen beteiligt sind, weckt dies Begehrlichkeiten, Gier und Neid [10]. 
В этом смысле слово leider проявляет некоторое сходство с модальными словами с эпистемической и эвиденциальной семантикой. Аналогичная ситуация может иметь место со словами erfreulicherweise и bedauerlicherweise. Тем не менее, их существенное отличие от слова leider заключается в способности образовывать словосочетания в комбинации с прилагательными и наречиями, как это можно проследить на следующем примере:

(39) Erfreulicherweise niedrig war die Zahl der Schulentlassenen ohne Hauptschulabschluss [10].

Это позволяет заключить, что слова erfreulicherweise и bedauerlicherweise целесообразно отнести к разряду не модальных слов, а наречий.

\section{3. Результаты исследования и их обсуждение}

Модальные слова представляют собой дифференцированный класс слов. Различия проявляются в видах модальных значений и их синтаксических свойствах, причём модальная семантика влияет на синтаксические свойства модальных слов.

Синтаксические свойства модальных слов определялись по их способности быть в скопусе эпистемических маркеров, фактивных предикатов и условных операторов. Модальные слова с эпистемической (vielleicht, vermutlich) и с эвиденциальной семантикой, предполагающей, что в роли источника выступает говорящий (scheinbar, anscheinend), не могут иметь места в придаточных условия и объектных придаточных с фактивными предикатами в главной части. Это свидетельствует о том, что они не влияют на пропозициональное содержание высказывания и относятся к сфере пропозициональной установки. Модальное слово angeblich способно иметь место в придаточных условия - это свидетельствует о возможном его вхождении в пропозицию, поскольку оно указывает на некий внешний источник информации. Слово с оценочной семантикой leider не способно быть в скопусе фактивного предиката и эпистемического маркера, а также не всегда может иметь место в условном придаточном. На основании перечисленных синтаксических и семантических характеристик можно предложить следующую дифференциацию модальных слов немецкого языка:

i. Модальные слова с эпистемической семантикой (vermutlich, vielleicht, höchstwahrscheinlich);

ii. Модальные слова с эвиденциальной семантикой, указывающие на говорящего как на источник информации (scheinbar, anscheinend);

iii. Модальные слова, которые способны кодировать алетическую модальность (wirklich и tatsächlich) и которые можно рассматривать как маркеры фокуса контраста;

iv. Отдельную группу образуют модальные слова:

- angeblich с эвиденциальной семантикой, поскольку оно указывает исключительно на 3-е лицо как на источник информации;

- wahrscheinlich, способное выражать несколько модальных значений: оно способно иметь эпистемическую или эвиденциальную или алетическую и эвиденциальную семантику;

- leider, передающее эмоциональное состояние говорящего.

В целом можно заключить, что класс модальных слов неоднороден. Общий признак данного класса слов - маркировка отношения говорящего к содержанию высказывания. Синтаксические свойства модальных слов зависят от того, входят ли они в модализованную пропозицию или являются маркерами пропозициональной установки.

(C) Аверина А.В., 2021

\section{Список литературы}

1. Аверина А.В. Модальный синтаксис немецкого языка / А.В. Аверина. М.: ИИУ МГОУ, 2019. 144 с.

2. Булыгина Т.В. Языковая концептуализация мира (на материале русской грамматики) / Т.В. Булыгина, А.Д. Шмелев. М.: Языки русской культуры, 1997. 576 с.

3. Вольф Е.М. Функциональная семантика оценки. М.: Либроком, 2020. 278 с. 
4. Зиндер Л.Р. Современный немецкий язык / Л.Р. Зиндер, Т.В. Строева Т.В. 3-е изд. М.: Изд-во лит-ры на иностр. яз., 1957. $424 \mathrm{c}$.

5. Кривоносов А.Т. Система классов слов как отражение структуры языкового сознания (философские основы теоретической грамматики) / А.Т. Кривоносов. Москва-Нью-Йорк: ЧеРо, 2001. 846 с.

6. Москальская О.И. Грамматика немецкого языка. Теоретический курс / О.И. Москальская. М.: Издательство на иностранных языках, 1956. 394 с.

7. Плунгян В.А. Общая морфология: введение в проблематику / В.А. Плунгян. М.: Эдиториал УРСС, 2000. 384 с.

8. Abraham W. Über Unhintergehbarkeiten in der modernen Modalitätsforschung / W. Abraham // Modalität und Evidentialität. Trier: Focus, 2011. S. 125-147.

9. Admoni W. Der deutsche Sprachbau / W. Admoni. М.: Просвещение, 1986. 336 c.

10. DECOW 16 A. [Электронный pecypc].- URL: http: www.webcorpora.org (дата обращения: 11.06.2020 - 12.07.2020).

11. Fritz Th. Zu Semantik, Textbezug und Pragmatik der konditional basierten Konnektoren / Th. Fritz // Worte über Wörter. Festschrift zu Ehren von Elke Ronneberger-Sibold. Tübingen: Stauffenburg Verlag, 2018. S. 115-130.

12. Helbig G. Deutsche Grammatik. Ein Handbuch für den Ausländerunterricht / G. Helbig, J. Buscha. Berlin, München, Wien, Zürich, New York, 2005. 654 S.

13. Höhle T.N. Über Verum-Fokus im Deutschen / T.N. Höhle // Informationsstruktur und Grammatik. Linguistische Berichte. Sonderheft 4. Opladen: Westdeutscher Verlag, 1992. S.112-141.

14. Hundt M. Epistemische Modalität im Deutschen / M. Hundt // Zeitschrift für germanistische Linguistik 31, 2003. S.343-381.

15. Kotin M. Modalitäten / M. Kotin // Zeitschrift des Verbandes polnischer Germanisten. Wydawnitwo Uniwersytetu Jagiellonskiego: Jagiellonian University Press, 2012. S.140-158.

16. Leiss E. Drei Spielarten der Epistemizität, drei Spielarten der Evidentialität und drei Spielarten des Wissens / E. Leiss // Modalität: Epistemik und Evidentialität bei Modaladverb, Adverb, Modalpartikel und Modus. Tübingen: Stauffenburg, 2009. S. 3-24.

17. Leiss E. Lexikalische versus grammatische Epistemizität und Evidentialität: Plädoyer für eine klare Trennung von Lexikon und Grammatik / E. Leiss // Modalität und Evidentialität [Fokus 37]. Trier: Wissenschaftlicher Verlag. S.149-169.

18. Lötscher A. Satzakzent und funktionale Satzperspektive im Deutschen / A. Lötscher. Tübingen: Niemyer, 1983. 285 S.

19. Lyons J. Semantik / J. Lyons. Bd. II, München, 1983. 508 S.

20. Pittner K. Adverbiale im Deutschen. Untersuchungen zu ihrer Stellung und Interpretation / K. Pittner. Tübingen: Stauffenburg Verlag, 1999. 390 S.

21. Remarque E.M. Drei Kameraden. Moskau: Verlag für fremdsprachige Literatur 1960. 585 S.

22. Zifonun G., Hoffmann L., Strecker B. Grammatik der deutschen Sprache. Berlin et al.: De Gruyter, 1997. 2570 S.

\section{References}

1. Averina, A.V. Modal'ny sintaksis nemetskogo iazyka [Modal syntax of the German language] / A.V. Averina. M.: IIU MGOU, 2019. $144 \mathrm{~s}$

2. Bulygina, T.V. Iazykovaia kontseptualizatsiia mira (na materiale russkoi grammatiki) [Linguistic conceptualization of the world (based on the material of Russian grammar)] / T.V. Bulygina, A.D. SHmelev. M.: IAzyki russkoi kul'tury, 1997. 576 c.

3. Vol'f, E.M. Funktsional'naia semantika otsenki [Functional evaluation semantics]. /E.M. Vol'f. M.: Librokom, 2020. 278 s.

4. Zinder, L.R. Sovremenny nemetskiy iazyk [Modern German] / L.R. Zinder, T.V. Stroeva T.V. 3-e izd. M.: Izd-vo lit-ry na inostr. iaz., 1957. $424 \mathrm{~s}$.

5. Krivonosov, A.T. Sistema klassov slov kakotrazhenie struktury iazykovogo soznaniia (filosofskie osnovy teoreticheskoigrammatiki) [The system of the word ckasses as the reflection of the structure of linguistic consciousness (philosophical foundations of theoretical grammar)] / A.T. Krivonosov. Moskva-N’yu-Jork: CHeRo, 2001. $846 \mathrm{~s}$.

6. Moskal'skaya, O.I. Grammatika nemetskogo iazyka. Teoreticheskiy kurs [German Grammar. The theoretical course] / O.I. Moskal'skaya. M.: Izdatel'stvo na inostrannykh iazykakh, 1956. 394 S.

7. Plungyan, V.A. Obshchaia morfologiia: vvedenie v problematiku [General morphology: an introduction to the problematic] / V.A. Plungyan. M.: Editorial URSS, 2000. 384 c.

8. Abraham, W. Über Unhintergehbarkeiten in der modernen Modalitätsforschung [About Inevitability of modal studies] / W. Abraham // Modalität und Evidentialität. Trier: Focus, 2011. S. 125-147.

9. Admoni, W. Der deutsche Sprachbau [Language structure of German] / W. Admoni. M.: Prosveshchenie, 1986. $336 \mathrm{s.}$

10. DECOW 16 A. - www.webcorpora.org (accessed: 11.06.2020 - 12.07.2020).

11. Fritz, Th. Zu Semantik, Textbezug und Pragmatik der konditional basierten Konnektoren [On the semantics, text reference and pragmatics of the conditionally based connectors] / Th. Fritz // Worte über Wörter. Festschrift zu Ehren von Elke RonnebergerSibold. Tübingen: Stauffenburg Verlag, 2018. S. 115-130.

12. Helbig, G. Deutsche Grammatik. Ein Handbuch für den Ausländerunterricht [German Grammar. A handbook for the foreigners' course] / G. Helbig, J. Buscha. Berlin, München, Wien, Zürich, New York, 2005. 654 S.

13. Höhle, T.N. Über Verum-Fokus im Deutschen [About the Verum-Focus] / T.N. Höhle // Informationsstruktur und Grammatik. Linguistische Berichte. Sonderheft 4. Opladen: Westdeutscher Verlag, 1992. S. 112-141.

14. Hundt, M. Epistemische Modalität im Deutschen [Epistemic modality in German] / M. Hundt // Zeitschrift für germanistische Linguistik 31, 2003. S. 343-381.

15. Kotin, M. Modalitäten [Modalities] / M. Kotin // Zeitschrift des Verbandes polnischer Germanisten. Wydawnitwo Uniwersytetu Jagiellonskiego: Jagiellonian University Press, 2012. S. 140-158. 
16. Leiss, E. Drei Spielarten der Epistemizität, drei Spielarten der Evidentialität und drei Spielarten des Wissens [Three varieties of epistemicity, three varieties of evidentiality and three varieties of knowledge] / E. Leiss // Modalität: Epistemik und Evidentialität bei Modaladverb, Adverb, Modalpartikel und Modus. Tübingen: Stauffenburg, 2009. S. 3-24.

17. Leiss, E. Lexikalische versus grammatische Epistemizität und Evidentialität: Plädoyer für eine klare Trennung von Lexikon und Grammatik [Lexical versus grammatical epistemicity and evidentiality: A plea for a clear separation of lexicon and grammar] / E. Leiss // Modalität und Evidentialität [Fokus 37]. Trier: Wissenschaftlicher Verlag. S. 149-169.

18. Lötscher, A. Satzakzent und funktionale Satzperspektive im Deutschen [Sentence accent and functional sentence perspective in German] / A. Lötscher. Tübingen: Niemyer, 1983. 285 S.

19. Lyons, J. Semantik [Semantics] / J. Lyons. Bd. II, München, 1983. 508 S.

20. Pittner, K. Adverbiale im Deutschen. Untersuchungen zu ihrer Stellung und Interpretation [Adverbials in German. Investigations into their position and interpretation] / K. Pittner. Tübingen: Stauffenburg Verlag, 1999. 390 S.

21. Remarque, E.M. Drei Kameraden [Three comrades] / E.M. Remarque. Moskau: Verlag für fremdsprachige Literatur 1960. $585 \mathrm{~S}$.

22. Zifonun, G. Grammatik der deutschen Sprache [German Grammar] / G. Zifonun, L. Hoffmann, B. Strecker. Berlin et al.: De Gruyter, 1997.2570 S.

\section{Сведения об авторе:}

Аверина Анна Викторовна - доктор филологических наук, профессор кафедры германской филологии МГОУ (Россия, Москва). Сфера научных и профессиональных интересов: грамматика немецкого языка, сравнительная типология немецкого и русского языков. E-mail: av.averina@mgou.ru

\section{About the author:}

Anna V. Averina - Doctor in Philological Sciences, Professor at the Department of German philology, Moscow Region State University; spheres of research and professional interest: grammar of German, typology of German and Russian. E-mail: av.averina@mgou.ru 